Relations industrielles

Industrial Relations

\title{
St-Onge, Sylvie, Michel Audet, Victor Haines et André Petit, Relever les défis de la gestion des ressources humaines
}

\section{Tania Saba}

Volume 54, numéro 4, 1999

URI : https://id.erudit.org/iderudit/051275ar

DOI : https://doi.org/10.7202/051275ar

Aller au sommaire du numéro

Éditeur(s)

Département des relations industrielles de l'Université Laval

ISSN

0034-379X (imprimé)

1703-8138 (numérique)

Découvrir la revue

Citer ce compte rendu

Saba, T. (1999). Compte rendu de [St-Onge, Sylvie, Michel Audet, Victor Haines et André Petit, Relever les défis de la gestion des ressources humaines]. Relations industrielles / Industrial Relations, 54(4), 818-820.

https://doi.org/10.7202/051275ar

Tous droits réservés @ C Département des relations industrielles de l'Universite Laval, 1999
Ce document est protégé par la loi sur le droit d'auteur. L'utilisation des services d'Érudit (y compris la reproduction) est assujettie à sa politique d'utilisation que vous pouvez consulter en ligne.

https://apropos.erudit.org/fr/usagers/politique-dutilisation/ 


\section{Recensions}

\section{Book Reviews}

\section{Relever les défis de la gestion des ressources humaines}

par Sylvie ST-ONGE, Michel AUDET, Victor HAINES et André PETIT, Montréal :

G. Morin, 1998, 701 p., ISBN 2-89105-683-3.

Relever les défis de la gestion des ressources humaines est un livre de base en gestion des ressources humaines destiné à un large public composé à la fois d'étudiants et de gestionnaires qui désirent acquérir des connaissances générales ou spécialisées en gestion des ressources humaines.

Construit autour de 16 chapitres, cet ouvrage reprend les activités de base de la gestion des ressources humaines, dont la planification, la dotation, la formation, la gestion des carrières, l'évaluation du rendement, la rémunération et l'organisation du travail, qu'il présente d'une manière originale. En effet, les auteurs annoncent six parties qui correspondent aux défis inhérents à la gestion des ressources humaines, soit les défis du partenariat, du renouvellement, de la compétence, du rendement, des conditions de travail et de la légitimité. Les différentes activités qui sont examinées sont exprimées comme étant des réponses à ces défis. Sans remettre en question les activités de base, cette perspective distingue cet ouvrage et témoigne d'une façon nouvelle d'aborder la gestion des ressources humaines.

Un premier chapitre d'introduction tente de résumer les principaux défis qui guettent la gestion des ressources humaines. Les auteurs rappellent les principales définitions, l'historique de la fonction, les différentes approches utilisées pour mieux comprendre la discipline et les rôles des différents intervenants dans le milieu du travail.
La première partie du livre présente les défis du partenariat en gestion des ressources humaines et englobe deux chapitres. Le premier examine essentiellement le partage des rôles entre les différents acteurs impliqués dans la gestion du personnel. Un second chapitre, de nature plus juridique, relate les différentes lois et les règlements qui encadrent les relations en emploi. Les structures syndicales y sont également traitées.

La deuxième partie du livre examine les défis du renouvellement en gestion des ressources humaines en présentant, dans un premier temps, la nature des changements qui y contribuent. Dans un chapitre subséquent, la planification des ressources humaines dans ce nouveau contexte est révélée comme étant un moyen pour réaliser la cohérence de la gestion des ressources humaines et lui donner une orientation.

La troisième partie de l'ouvrage présente les activités de dotation, de formation et de gestion des carrières comme étant des réponses aux défis de la compétence. Le chapitre sur la dotation traite des questions d'embauche, comme les moyens à mettre en œuvre pour susciter des candidatures, pour procéder à une sélection efficace et pour favoriser l'intégration de la personne recrutée. Le chapitre sur la formation traite des processus et de l'encadrement juridique du développement des compétences dans les organisations. Le dernier chapitre de cette troisième partie présente le système, les 
déterminants, les caractéristiques et les pratiques de gestion des carrières.

Quant à la quatrième partie de l'ouvrage, intitulée les défis du rendement, elle englobe la gestion du rendement au travail, sa reconnaissance et l'intervention auprès des employés difficiles. Présenté sous forme de principes à respecter, le chapitre traitant de la gestion du rendement au travail énumère les pratiques de gestion du rendement qu'il conviendrait d'implanter afin d'assurer le succès de la démarche d'évaluation. Le chapitre sur la reconnaissance du rendement au travail fait connaître les différentes formes de récompenses et de gratification des employés. À cette occasion, les questions de rémunération ayant trait aux régimes collectifs et aux régimes incitatifs sont abordées, mais la question salariale est étudiée ultérieurement, soit dans la partie sur les conditions de travail. Pour terminer cette quatrième partie du livre, un chapitre est consacré au thème des employés difficiles et offre aux lecteurs des moyens d'intervention auprès des employés qui vivent des problèmes professionnels. Le chapitre se termine par un examen rapide des programmes d'aide aux employés et aborde de manière élaborée l'encadrement juridique des mesures disciplinaires.

Les défis des conditions de travail sont examinés à travers la gestion de la rémunération et des avantages sociaux, l'organisation du travail, la négociation et l'administration des conventions collectives ainsi que la promotion de la santé et sécurité au travail. Le chapitre portant sur la rémunération retient particulièrement l'attention grâce aux nombreux systèmes et techniques qui y sont présentés. Un développement judicieux est réservé à l'équité salariale, une question d'actualité. L'examen de la gestion des avantages sociaux est également à propos. Le chapitre consacré à l'organisation du travail évoque l'évolution des structures et des systèmes organisationnels avant d'aborder les nouveaux modèles d'organisation du travail. Ce chapitre fait état des expériences d'organisation du travail dans d'autres pays et aborde les choix stratégiques qui s'imposent aux employeurs afin d'adopter des modèles d'organisation du travail. Les chapitres portant sur la négociation collective et la promotion de la santé et sécurité au travail donnent un aperçu adéquat et à jour des questions examinées.

Finalement, le demier défi, soit celui de la légitimité de la gestion des ressources humaines, traite de la question de l'évaluation de l'efficacité de la fonction et de son renouvellement. Dans ce chapitre, sont présentés la définition, le modèle, les raisons et les diverses approches d'évaluation de la fonction et des pratiques de gestion des ressources humaines. L'ouvrage se termine en retenant les tendances que les auteurs considèrent les plus marquantes de la prochaine décennie.

Rédiger un ouvrage en gestion des ressources humaines constitue de nos jours un défi de taille. Rallier les modèles stratégiques et opérationnels sans succomber à la tentation de présenter des modèles conceptuels qui risquent d'éloigner le lecteur de la réalité organisationnelle est, certes, souvent difficile à réaliser. À cela, il faut ajouter que la complexité des différentes activités et la profusion des aspects techniques ajoutent à la difficulté de fournir au lecteur un instrument de travail qui rassemble en même temps les nouvelles tendances et les moyens pour parvenir à une gestion efficace des ressources humaines.

La première force du livre est d'avoir su adopter une approche d'actualité qui se prête bien à l'orientation stratégique de la gestion des ressources humaines. En effet, cet ouvrage qui intègre les activités de gestion des ressources humaines aux différents défis environnementaux et organisationnels a réussi à ne pas perdre de vue cet aspect important.

Étant donné que le livre traite principalement des défis en gestion des res- 
sources humaines, on se serait attendu à ce que les activités soient systématiquement abordées selon une approche stratégique. À cet égard, les auteurs ont préféré privilégier, dans certains cas, une approche plus opérationnelle. Sur les aspects plus administratifs de la gestion des ressources humaines, certains chapitres auraient gagné à développer davantage les moyens techniques qui accompagnent l'implantation des différentes activités.

Cela dit, la plupart des chapitres sont bien documentés. La bibliographie récente atteste de l'actualité des différents propos abordés dans les chapitres et constitue une source de références supplémentaires. À l'occasion, certaines adresses de sites sur internet sont offertes au lecteur. Les maintes mises en situation et illustrations ainsi que les différents encadrés permettent à la fois de couvrir des aspects plus pratiques et de fixer les idées principales qui se dégagent du texte. Notons également que deux ou trois cas terminent chacun des chapitres. Ces cas, qui sont succincts, constituent une source d'apprentissage appréciée des étudiants puisqu'ils leur permettent d'appliquer les notions apprises dans le chapitre. Aussi, cet ouvrage nous fait bénéficier des travaux de recherche des auteurs qui y ont collaboré.

En somme, ce livre demeure un ouvrage de base intéressant qui examine avec originalité la gestion des ressources humaines. Il permet de faire le point sur les principales activités et illustre astucieusement un domaine qui, à certains égards, peut paraître très normatif, soit celui de la gestion des ressources humaines.

\section{TANIA SABA \\ École de relations industrielles Université de Montréal}

\section{Sociologie du travail et gestion des ressources humaines}

par Michel DE COSTER avec la collaboration d'Annie CORNET et de Christine

DELHAYE, Bruxelles : DeBoeck Université, 1999, 3édition [1987], 344 p., ISBN

2-8041-3122-X.

Il s'agit de la troisième édition d'un ouvrage fort apprécié paru en 1987 sous le titre Sociologie du travail et gestion $d u$ personnel. Cette troisième édition est significativement revue et augmentée compte tenu du contexte de l'activité socio-économique (chômage chronique, aménagement du temps de travail, informatisation des organisations) et du désir de l'auteur de rendre son ouvrage accessible à un public plus étendu que celui des étudiants et des chercheurs, en s'adressant notamment aux gestionnaires des ressources humaines dans les entreprises.

Cet ouvrage n'est pas qu'un livre d'initiation parmi d'autres. Il se distingue des autres à la fois par son intention et par son écriture. L'intention est originale: éclairer la gestion des ressources humaines par la perspective sociologique du travail pour mieux l'ancrer dans la réa- lité sociale des organisations. Pour De Coster, il est urgent de faire comprendre les enjeux réels des techniques de gestion trop souvent " plaquées sur des tissus sociaux dont on n'a retenu ni la diversité ni l'originalité ". C'est précisément parce que la gestion des ressources humaines présente un caractère instrumental et normatif qu'il lui est interdit de faire l'économie de la description et de l'observation :

"Avant d'agir sur la réalité organisationnelle, il convient de la connaître, à travers sa culture, ses valeurs, ses traditions, la configuration de ses groupes sociaux réputés formels ou informels, ses jeux et ses enjeux de pouvoir. Au surplus, méconnaître l'analyse sociologique consacrerait dans la réalité des faits, l'échec fréquent des techniques de gestion greffées sur des tissus sociaux très divers. Pour avoir méconnu cette idée capitale 